\title{
Temporizing amplatzer closure of an aorto-enteric fistula associated with a blind aortic stump via a translumbar approach
}

\author{
S. Keisin Wang, Justin R. King, Ashley R. Gutwein, Raghu L. Motaganahalli, Andres Fajardo, Gary W. \\ Lemmon
}

Division of Vascular Surgery, Department of Surgery, Indiana University School of Medicine, Indianapolis, IN 46202, USA.

Correspondence to: Dr. S. Keisin Wang, Division of Vascular Surgery, Department of Surgery, Indiana University School of Medicine, 1801 Senate BIvd MPC\# 2-3500, Indianapolis, IN 46202, USA. E-mail: wangkei@iupui.edu

How to cite this article: Wang SK, King JR, Gutwein AR, Motaganahalli RL, Fajardo A, Lemmon GW. Temporizing amplatzer closure of an aorto-enteric fistula associated with a blind aortic stump via a translumbar approach. Vesse/ P/us 2018;2:3.

http://dx.doi.org/10.20517/2574-1209.2017.38

Received: 13 Dec 2017 First Decision: 17 Jan 2018 Revised: 18 Jan 2018 Accepted: 27 Jan 2018 Published: 7 Feb 2018

Science Editor: Aaron S. Dumont Copy Editor: Jun-Yao Li Production Editor: Huan-Liang Wu

\begin{abstract}
We present a case of an aorto-enteric fistula (AEF) with chronic, persistent bleeding from a blind aortic stump managed by endovascular means. This novel approach may have extended the life of a patient who would otherwise have been subject to a high perioperative morbidity or persistent bleeding and death. While our patient ultimately expired, we believe this technique can be considered for temporization in highly-selected patients.
\end{abstract}

Keywords: Aorto-enteric fistula, amplatzer vascular plug, endovascular treatment

\section{INTRODUCTION}

Aorto-enteric fistula (AEF) is a highly-morbid complication following aortic reconstruction. Although the traditionally accepted method of treatment is open surgical repair, alternative methods need to be considered in high operative-risk patients. We present a case of endovascular plug placement within a blind aortic stump for treatment of an AEF 16 years after an index aorto-bifemoral bypass (ABF). While the patient initially experienced resolution of his symptoms, this ultimately proved to be a temporizing measure as the patient eventually died of hemorrhagic shock related to AEF progression.

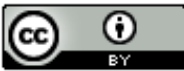

(C) The Author(s) 2018. Open Access This article is licensed under a Creative Commons Attribution 4.0 International License (https://creativecommons.org/licenses/by/4.0/), which permits unrestricted use, sharing, adaptation, distribution and reproduction in any medium or format, for any purpose, even commercially, as long as you give appropriate credit to the original author(s) and the source, provide a link to the Creative Commons license, and indicate if changes were made. 


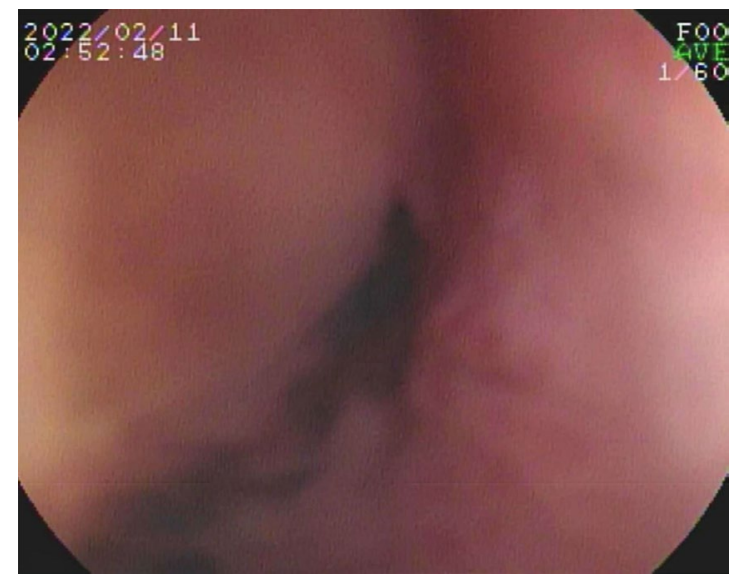

Figure 1. Double-balloon upper endoscopy demonstrated a mucosal defect in the 3rd portion of the duodenum suggestive of a sinus tract. This area was clipped to mark the anatomic location. Computed tomography angiogram following endoscopy demonstrated a diverticulum immediately adjacent to the clip suggestive of a small aorto-enteric fistula

\section{CASE REPORT}

A 75-year-old male was transferred to our facility for evaluation of intermittent gastrointestinal (GI) bleeding requiring over 20 units of packed red blood cells over the 6 months previous to admission. His past surgical history was significant for $\mathrm{ABF}$ bypass to treat and infrarenal abdominal aortic aneurysm in 2001. Three years following his initial operation, the patient developed a perigraft infection complicated by AEF treated by partial colectomy, end ileostomy, aortic graft explantation, ligation of the infrarenal aorta, and axillo-bifemoral bypass. The blind aortic stump was created two centimeters distal to the renal ostia.

The GI bleeding was initially evaluated at an outside facility with multiple computed tomography angiograms (CTAs), capsule endoscopies, and direct upper/lower GI endoscopies. Following transfer to our facility, double-balloon endoscopy demonstrated a small mucosal defect suspicious for a sinus tract in the 3rd portion of the duodenum [Figure 1]. An endoclip was placed for as a reference point. Repeat CTA visualized the endoclip near an aortic diverticulum at the inferior border of the blind stump [Figure 2]. Because of his medical comorbidities (chronic kidney disease III, hypertension, anemia, frailty, multiple abdominal operations creating a hostile abdomen with left-sided ostomy) and patient preference, open surgery was ruled out as a treatment option. At this point, we elected to proceed with embolization of the distal aortic stump.

Under moderate sedation, the left radial artery was accessed. After heparin infusion, a long 5-F catheter was positioned in the aorta and a diagnostic aortogram was performed. Unfortunately, the brachial artery was of unsuitable size for the sheath required for our planned embolization. Using a "down the barrel" technique, an $18-\mathrm{G}$ trocar needle was advanced into the terminal aspect of the abdominal aortic stump via a translumbar approach. An 8-F sheath was inserted via the translumbar access, positioned in the abdominal aorta, and confirmed via angiography. A 22-mm Amplatzer II Vascular Plug (St. Jude Medical) was then deployed such that the plug spanned from the luminal aspect of the terminal abdominal aorta to the extraluminal portion of the aortic stump to prevent migration. Through the radial artery, multiple Ruby (Penumbra) coils were deployed past the vascular plug to thrombose the residual stump [Figure 3].

The patient had an uneventful postoperative course and was discharged 2 days later on lifelong oral antibiotics. At a 5-week postoperative visit, imaging demonstrated continued thrombosis of the aortic stump, decreased periaortic soft tissue swelling, and no extravasation of contrast [Figure 4]. The patient stated that his GI bleeding had completely resolved. 


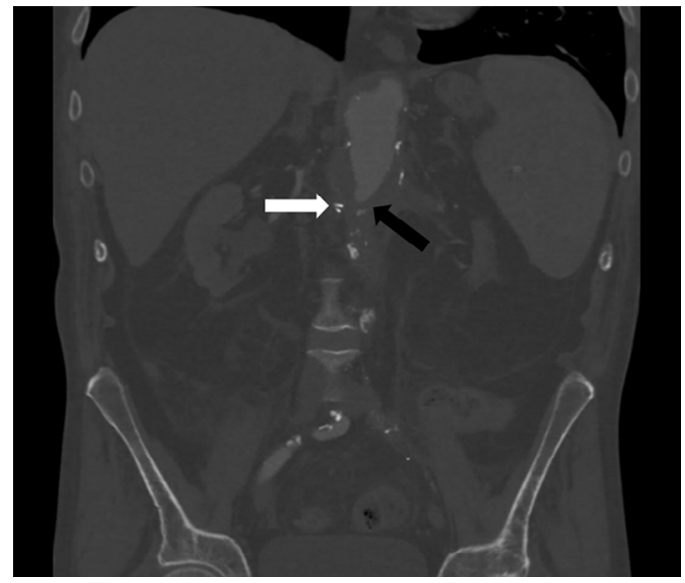

Figure 2. After placement of a metallic clip in the duodenum at the visualized lesion, a computed tomography angiogram demonstrated a diverticulum of the aortic stump (black arrow) near the duodenal clip (white arrow) concerning for another aorto-enteric fistula
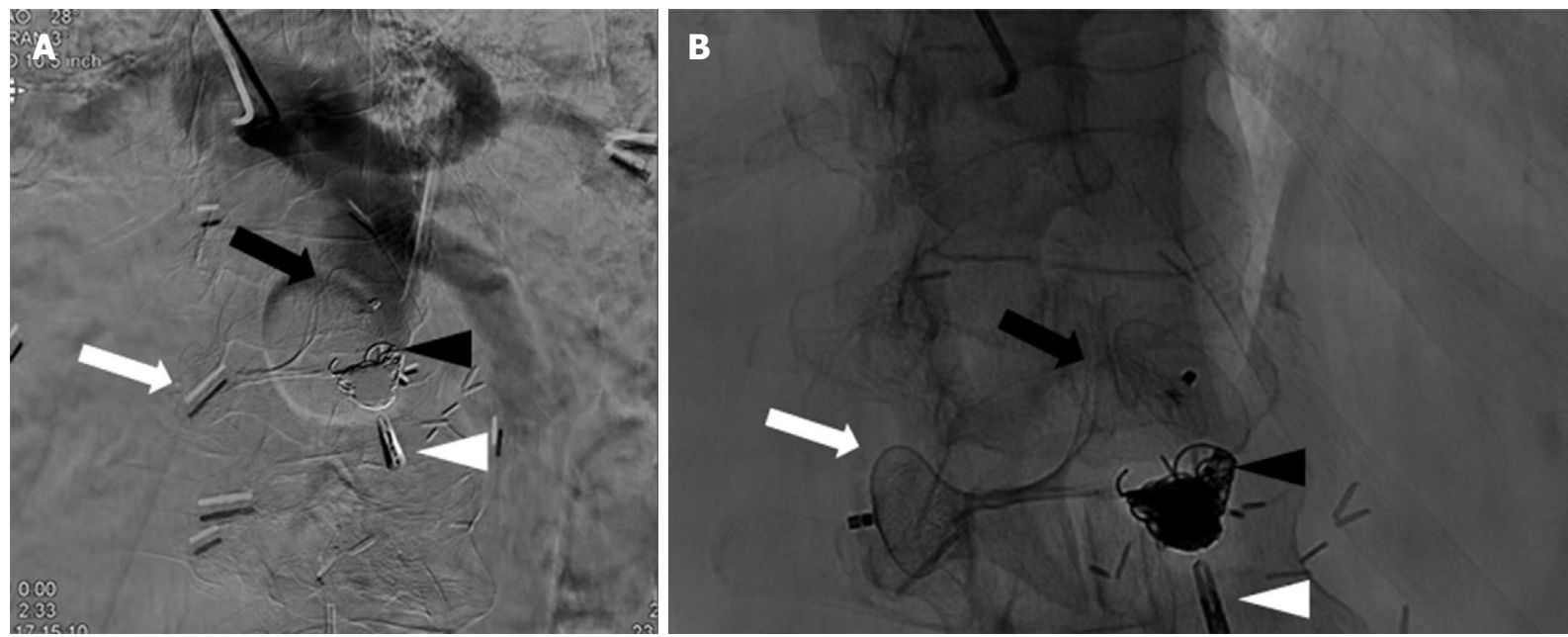

Figure 3. Digital subtraction angiography (A) and fluoroscopy (B) demonstrated placement of the Amplatzer II Vascular Plug so that 2/3 of the plug remained intraluminally (black arrow) in the aortic stump while 1/3 remained in the periaortic space (white arrow). Microcoils were deployed distal to the intraluminal plug to further assist in stump thrombosis (black arrowhead). The close association between the aortic stump and the duodenum is demonstrated by the endoclip placed at the time of double-balloon endoscopy (white arrowhead)

Approximately 14 weeks after our procedure, the patient presented with recurrent GI bleeding. The AEF was again identified by endoclip on upper GI endoscopy. The patient underwent further coil placement within the aortic stump via the radial artery. Following this reintervention, he was discharged home without repeat bleeding. Unfortunately, he proceeded to present in similar fashions 2 additional times; the first was managed with endoscopic clipping. However, on his final presentation 5 months postoperatively, the decision was made to proceed with definitive surgical ligation of the aortic stump given multiple failed endovascular and endoscopic interventions. On the day prior to his scheduled surgery, the patient developed acute hemorrhagic shock identified via his nasogastric tube. Unfortunately, he became hemodynamically unstable, was emergently intubated, and initiated on a massive transfusion protocol. After discussion with the patient's family, code status was changed to "do not resuscitate", comfort care was initiated, and he ultimately expired.

\section{DISCUSSION}

$\mathrm{AEF}$ is a rare and highly morbid complication following aortic intervention with an incidence of approximately $1 \%{ }^{[1]}$. In the aortic reconstruction patient, it is thought that pathogenesis is related to 


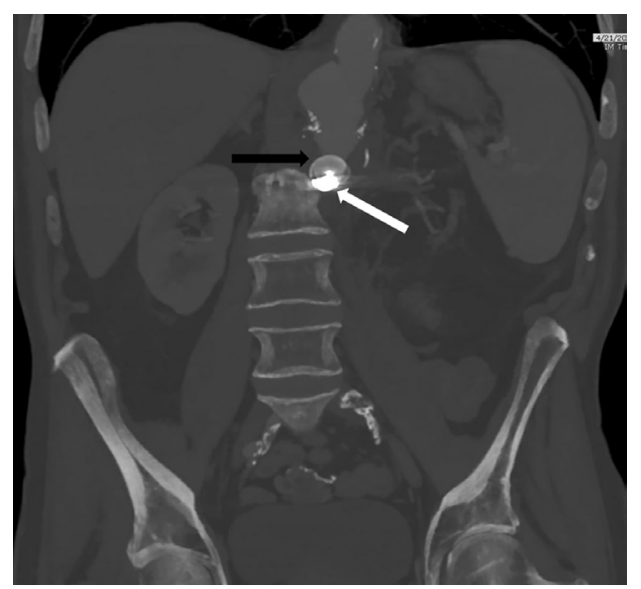

Figure 4. Five weeks post-operatively, the patient remained bleed-free without leukocytosis or fever. Computed tomography angiogram demonstrated improvement in peri-aortic inflammation and stable positioning of the intraluminal vascular plug (black arrow) and the microcoils (white arrow)

instrumentation, enteric contact with aortic wall or graft, and persistent inflammation ${ }^{[2-4]}$. Spontaneous rupture of the aorta through the fistulous connection can present as subclinical, persistent bleeding, or life threatening exsanguination and carries a mortality rate approaching $100 \%$ if left untreated ${ }^{[5,6]}$.

Patients developing AEF may prove to be difficult to diagnose as they tend to present with episodic bleeding. Therefore, a high index of suspicion must be maintained. Other clinical manifestations may include abdominal pain, palpable abdominal mass, and nausea ${ }^{[2]}$. However, these symptoms are either vague or often absent. Our patient presented with episodic bleeding without other accompanying symptoms for 6 months and underwent multiple CTAs and endoscopies before he was diagnosed. This illustrates well the difficulty in making the diagnosis and locating the site of pathology.

High-quality evidence regarding outcomes after treatment of AEFs are severely lacking ${ }^{[5]}$. One of the larger experiences was published by Armstrong et al. ${ }^{[7]}$ in 2005. In this study, the authors described their experience with secondary AEFs in 29 patients. The most common procedures performed were excision with extra-anatomic bypass including axillo-femoral and cross-femoral bypass grafting $(n=25)$, aortofemoral grafting with additional lower-limb bypasses $(n=2)$, and in situ reconstruction with rifampinsoaked Dacron $(n=2)$. Perioperative mortality in those who received an operation at 30 -day was $21 \%{ }^{[7]}$.

A European meta-analysis of both endovascular and open intervention for secondary AEFs was published in 2016 by Kakkos et al. ${ }^{[8]}$. The authors included 98 patients who received endovascular intervention along with 725 open repairs from 1999 to 2015. In-hospital mortality was 7.1\% in the endovascular group compared to the $33.9 \%$ in the open repair cohort, though this would be expected given the intent of each treatment modality, specifically palliation versus definitive repair. Interestingly, there was no difference in recurrence between the two treatment modalities. While the early survival benefit dampened during follow-up, it continued to remain significant. Not surprisingly, late sepsis was twice as high in the endovascular group compared to open repair at two years postoperatively ( $42 \% v s$. $19 \%$ ). The authors conclude that endovascular repair is associated with early benefit which is lost over time; therefore, they argue for staged repair with eventual conversion to in situ vein grafting in selected patients ${ }^{[8]}$.

Based on our previous experience and the established literature, we did not believe our patient would have survived an open operation and therefore reserved open repair initially. He had multiple abdominal procedures stemming from his original aortic reconstruction which resulted in takebacks, ostomies, and 
intraabdominal abscesses. Additionally, he suffered from frailty, malnutrition, and chronic kidney disease. After discussion regarding his treatment options, he was adamant on intervention to stop the GI bleeding. Therefore, an endovascular intervention was attempted as a palliative procedure. Although our patient initially demonstrated cessation of his GI bleeding following endovascular plug deployment, this success was short-lived and the patient experienced recurrence of his symptoms with ultimate exsanguination and death.

\section{DECLARATIONS}

\section{Authors' contributions}

Concept: Wang SK

Design, literature search, manuscript preparation, manuscript editing, review: Wang SK, King JR, Gutwein AR, Motaganahalli RL, Fajardo A, Lemmon GW

\section{Financial support and sponsorship}

None.

\section{Conflicts of interest}

There are no conflicts of interest.

\section{Patient consent}

Written consent was obtained from the family of the deceased and is available upon request.

\section{Ethics approval}

Not applicable.

\section{Copyright}

(c) The Author(s) 2018.

\section{REFERENCES}

1. Song Y, Liu Q, Shen H, Jia X, Zhang H, Qiao L. Diagnosis and management of primary aortoenteric fistulas--experience learned from eighteen patients. Surgery 2008;143:43-50.

2. Duncan JR, Renwick AA, Mackenzie I, Gilmour DG. Primary aortoenteric fistula: pitfalls in the diagnosis of a rare condition. Ann Vasc Surg 2002;16:242-5.

3. Sweeney MS, Gadacz TR. Primary aortoduodenal fistula: manifestation, diagnosis, and treatment. Surgery 1984;96:492-7.

4. van Olffen TB, Knippenberg LH, van der Vliet JA, Lastdrager WB. Primary aortoenteric fistula: report of six new cases. Cardiovasc Surg 2002;10:551-4.

5. Saers SJ, Scheltinga MR. Primary aortoenteric fistula. Br J Surg 2005;92:143-52.

6. Voorhoeve R, Moll FL, de Letter JA, Bast TJ, Wester JP, Slee PH. Primary aortoenteric fistula: report of eight new cases and review of the literature. Ann Vasc Surg 1996;10:40-8.

7. Armstrong PA, Back MR, Wilson JS, Shames ML, Johnson BL, Bandyk DF. Improved outcomes in the recent management of secondary aortoenteric fistula. J Vasc Surg 2005;42:660-6.

8. Kakkos SK, Bicknell CD, Tsolakis IA, Bergqvist D; Hellenic Co-operative Group on Aortic Surgery. Management of secondary aortoenteric and other abdominal arterio-enteric fistulas: a review and pooled data analysis. Eur J Vasc Endovasc Surg 2016;52:770-86. 